\title{
Does the Addition of Manual Therapy Approach to a Cervical Exercise Program Improve Clinical Outcomes for Patients with Chronic Neck Pain in Short- and Mid-Term? A Randomized Controlled Trial
}

\author{
Jacobo Rodríguez-Sanz ${ }^{1, *} \mathbb{C}$, Miguel Malo-Urriés ${ }^{2,3}$, Jaime Corral-de-Toro ${ }^{2,3}$,

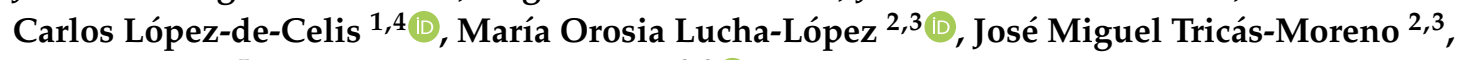 \\ Ana I Lorente ${ }^{5}$ and César Hidalgo-García ${ }^{2,3}$ (D) \\ 1 Faculty of Medicine and Health Sciences, Universitat Internacional de Catalunya, \\ 08195 Sant Cugat del Vallès, Spain; carlesldc@uic.es \\ 2 Faculty of Health Sciences, Universidad de Zaragoza, 50009 Zaragoza, Spain; malom@unizar.es (M.M.-U.); \\ jaimecorral.fisio@gmail.com (J.C.-d.-T.); orolucha@unizar.es (M.O.L.-L.); jmtricas@unizar.es (J.M.T.-M.); \\ hidalgo@unizar.es (C.H.-G.) \\ 3 Physiotherapy Research Unit, Universidad de Zaragoza, 50009 Zaragoza, Spain \\ 4 Fundació Institut Universitari per a la recerca a l'Atenció Primària de Salut Jordi Gol i Gurina, \\ 08007 Barcelona, Spain \\ 5 Impact Laboratory, Aragón Institute of Engineering Research (I3A), Universidad de Zaragoza, \\ 50018 Zaragoza, Spain; analorente@unizar.es \\ * Correspondence: jrodriguezs@uic.es; Tel.: +34-636-13-67-89
}

Received: 20 July 2020; Accepted: 2 September 2020; Published: 10 September 2020

\begin{abstract}
Chronic neck pain is one of today's most prevalent pathologies. The International Classification of Diseases categorizes four subgroups based on patients' associated symptoms. However, this classification does not encompass upper cervical spine dysfunction. The aim is to compare the short- and mid-term effectiveness of adding a manual therapy approach to a cervical exercise protocol in patients with chronic neck pain and upper cervical spine dysfunction. Fifty-eight subjects with chronic neck pain and upper cervical spine dysfunction were recruited (29 = Manual therapy + Exercise; 29 = Exercise). Each group received four 20-min sessions, one per week during four consecutive weeks, and a home exercise regime. Upper flexion and flexion-rotation test range of motion, neck disability index, craniocervical flexion test, visual analogue scale, pressure pain threshold, global rating of change scale, and adherence to self-treatment were assessed at the beginning, end of the intervention and at 3- and 6-month follow-ups. The Manual therapy + Exercise group statistically improved short- and medium-term in all variables compared to the Exercise group. Four 20-min sessions of Manual therapy + Exercise along with a home-exercise program is more effective in the short- to mid-term than an exercise protocol and a home-exercise program for patients with chronic neck pain and upper cervical dysfunction.
\end{abstract}

Keywords: upper cervical spine; manual therapy; training; neck pain

\section{Introduction}

Chronic neck pain is one of the most prevalent pathologies nowadays, accounting for $14.6 \%$ of all musculoskeletal health problems [1]. It is estimated that $50 \%$ of all adults experience some kind of neck pain at some point each year [2].

Cervical exercise has been shown to be an effective treatment for neck pain [3,4]. A recent systematic review in patients with chronic neck pain concluded that multimodal training (exercises involving deep 
and superficial cervical muscles) is necessary to have beneficial effects on function and symptoms [5]. Some studies have considered if a manual therapy approach should be added or not to the cervical exercise protocol for neck pain $[4,6]$. These studies have been carried out in neck pain subgroups according to the International Classification of Diseases (neck pain with mobility deficits in the global cervical spine, neck pain with radiating pain "radicular pain", neck pain with movement coordination impairments, and neck pain with cervicogenic headache) and the results are prone to consider the effectiveness of cervical exercise [4,7]. However, there is a lack of clinical trials evaluating the effectiveness of the manual therapy approach on neck pain samples with upper cervical joint dysfunctions. Moreover, more than $60 \%$ of cervical axial rotation occurs in the upper cervical spine [8], a fundamental region for cervical function. Lack of mobility and symptoms arising from upper cervical joints are considered to be the main indication for upper cervical manual therapy approach. In addition, upper cervical dysfunction could limit the efficacy of cervical exercise in this sample of chronic neck pain patients. The effect of these treatments in a new subgroup of patients with chronic neck pain and upper cervical spine dysfunction is currently unknown $[4,9,10]$.

The hypothesis of this study is that adding an upper cervical spine manual therapy approach to a cervical exercise protocol is more effective in improving function and symptomatology than an exercise protocol in patients with chronic neck pain and upper cervical spine dysfunction.

The objective of this study aimed to compare the short- and mid-term effectiveness of adding a manual therapy approach to a cervical exercise protocol in patients with chronic neck pain and upper cervical spine dysfunction.

\section{Methods}

\subsection{Study Design}

A randomized, longitudinal (simple 1:1) controlled clinical trial was conducted using the Microsoft Excel program for the randomization by an external researcher. Consolidated Standards of Reporting Trial (CONSORT) guidelines were followed throughout the study. Assignments were placed in a concealed opaque envelope, and participants were randomly assigned to intervention groups. The design was carried out in collaboration with the University of Zaragoza, "Delicias Sur" Health Center of Zaragoza, and the OMT-E Clinical Center of Zaragoza, Spain (Clinicaltrials.gov number: NCT03670719; date of first registration 13 September 2018). This study was approved by the local ethics committee (Comité Ético de Investigación Clínica de Aragón "CEICA"; 13/2018). All research was performed in accordance with relevant guidelines/regulations, and informed consent was obtained from all participants. The subjects in the images in this manuscript gave consent for publication in an online open-access publication.

\subsection{Sample Size Calculation}

The sample size was calculated based on the outcomes of two studies, Dunning et al. 2012 [11] and Izquierdo-Pérez et al. 2014 [12]. The common standard deviation and the minimum differences to be detected between the groups were determined using the outcomes of these two previously mentioned studies [11,12]. The main variables used for sample size calculation of our study were the flexion-rotation test [11] and neck disability index [12], obtaining the highest number of subjects (26 subjects per group using neck disability index variable), making a total sample of at least 52 subjects. The sample size was calculated using the GRANMO 7.12 program, with a $\alpha$ risk of 0.05 , test two-side, a $\beta$ risk of 0.20 . For the neck disability index variable, we used an estimated common standard deviation of 6.8 [12] and a minimum expected difference of 5.8 [12], estimating a follow-up loss of $15 \%$.

\subsection{Subjects}

Fifty-eight volunteer subjects were recruited (17 men; 41 women), six patients more than the required sample size. This was the total number of patients referred by doctors over a four-week 
period. The inclusion criteria comprised: a medical diagnosis of chronic neck pain that persists for more than 3 months [6], a positive result in the flexion-rotation test (less than $32^{\circ}$ or an asymmetry of $10^{\circ}$ or more between sides) [13,14], a failure to pass stage $2(24 \mathrm{mmHg})$ of the craniocervical flexion test [15], hypomobility in one or more segments of C0-1, C1-2, C2-3 through manual assessment according to Zito et al. 2006 [16] and Kaltenborn (2012) [17], grade I-II in the classification of cervical pain [18], being over 18 years old, and having signed the informed consent. Exclusion criteria comprised: contraindications for manual therapy or exercise, having participated in a cervical exercise or manual therapy program in the last three months, presenting warning signs or having suffered a relevant neck trauma [19], an inability to maintain supine position, the use of pacemakers, an inability to perform a flexion-rotation test, language difficulties, and pending litigation or lawsuits [20].

\subsection{Measurements}

The primary outcome measures in this study were the neck disability index and flexion-rotation test. Secondary outcome measures were upper cervical flexion range of motion, pain intensity, craniocervical flexion test, cervical pressure pain threshold, global rating of change scale (GROC-Scale), and adherence to self-treatment scale.

Neck disability was measured using the neck disability index. The test-retest reliability of this questionnaire is excellent (ICC 0.97) and has been validated in the Spanish language [21].

Pain intensity was assessed on a visual analogue scale from 0 to $10 \mathrm{~cm}$ in length, with no intermediate point. Test-retest reliability is excellent (ICC 0.92) [22].

A Flexion-rotation test was used to measure the upper rotation, predominantly at C1-2. The methodology proposed by Hall et al. 2007 [13] was followed. The subject was in the supine position, and the evaluator passively moved the patient's cervical spine to its maximum flexion and then rotated the head to the right and left side with the occiput resting against the evaluator's abdomen. The movement stopped at whichever situation occurred first, either the subject presented symptoms, or a firm end feel was encountered [13,23]. A CROM device (floating compass; Plastimo Airguide, Inc, Buffalo Groove, IL, USA) was used, and three measurements were taken for each rotation, with the result being the mean of the three measurements [20]. The range of motion to the more restricted $(+)$ and less restricted (-) rotation was considered. Flexion-rotation test reliability is between $0.93-0.96[24,25]$ and has excellent validity [26].

Active mobility of upper cervical spine flexion was measured in the standing position using a CROM device [27].

The craniocervical flexion test was used to measure the isolated activation of the deep flexor muscles. A Stabilizer Pressure Biofeedback Unit (Chattanooga, TN, USA) was used to measure this test. The activation and resistance of the deep cervical flexors were evaluated in five progressive pressure increases of $2 \mathrm{mmHg}$ up to a maximum of $30 \mathrm{mmHg}$. When the patient reached a level three times, he or she passed to the next level [28]. Test-retest reliability is excellent 0.98 ICC [29].

The cervical pressure pain threshold was measured using a digital algometer (Somedic AB Farsta, Somedic SenseLab AB, Sösdala, Sweden) with a round surface area of $1 \mathrm{~cm}^{2}$. Pressure was applied at a speed of $1 \mathrm{~kg} / \mathrm{cm}^{2} / \mathrm{s}$ perpendicular to the skin. With the subject supine, the pressure pain threshold was assessed over three points bilaterally: first metacarpal joint, C2-3 zygapophyseal joint, and suboccipital muscles. Patients were instructed to press the button of the digital algometer at the exact moment the sensation of pressure changed to pain. The mean of three trials was calculated over each point and used for analysis. Pressure pain threshold measurements have a high reliability (ICC $=0.92-0.99)[23,30,31]$.

The GROC-Scale was used to measure the personal self-perceived improvement that the patient had experienced [32,33]. The GROC-Scale is considered to be an efficient way to score patients' perceived clinical change [34]. The test-retest reliability of the GROC-Scale is excellent (ICC $=0.90)$ [35].

A scale of adherence to self-treatment was designed to discover the frequency that patients did their exercises at home. Patients were asked to choose among the following answers: "I have done the 
exercises every day, I have done the exercises 4-6 days a week, I have done the exercises 1-3 days a week, I have done the exercises less than 1 day a week, or I have not done them".

One clinical researcher performed all the treatment for all patients and another clinical researcher, with training in evaluation and more than 5 years' clinical experience, took all the clinical measurements for all patients before (T0), at the end of the intervention (T1), after 3 months (T2) and after 6 months (T3). This researcher remained blinded to each patient's assignment group throughout the process. Study participation was then complete, and researchers proceeded with an individualized treatment approach.

\subsection{Intervention}

The intervention was administered individually in the facilities of the Universidad de Zaragoza. Participants in both groups received one 20-min session once a week for four consecutive weeks. The treatment was applied by a researcher with more than 5 years' experience in physical therapy. A weekly video call was made for all patients to monitor their adherence to these recommendations during the home exercise program. This home exercise program was performed throughout the study.

\subsection{Exercise Group}

The exercise program was developed according to Fernández-de-las-Peñas et al. 2013 [36] and Jull et al. 2002 [37]. This exercise progression includes contraction of deep neck flexor muscles (Figure 1) and global muscles of the neck.
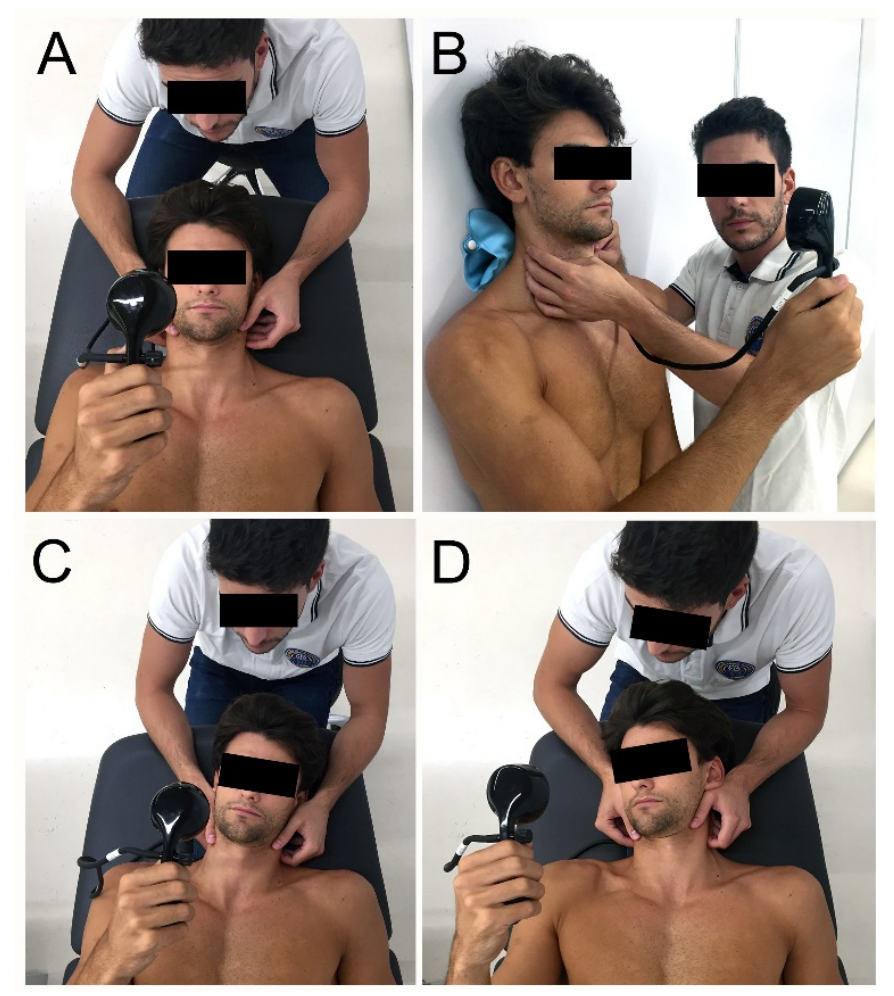

Figure 1. Contraction of deep neck flexor muscle progression. (A) Bilateral contraction in supine; (B) Bilateral contraction in standing; (C) Unilateral contraction with cervical inclination in supine; (D) Unilateral contraction with cervical rotation in supine.

An exercise program was carried out for one day a week for four weeks. Each exercise session lasted $20 \mathrm{~min}$ and was composed of two blocks of 10 repetitions, holding each exercise for $10 \mathrm{~s}$, with a $40 \mathrm{~s}$ rest between each repetition, and two minutes between blocks [36]. 
After T0 assessments, patients started with the first treatment session performing cervical stabilization exercises and were taught to perform the contraction of deep neck flexor muscle activity with the help of the Stabilizer Pressure Biofeedback Unit (Chattanooga, TN, USA) [28,38]. A progression was also added in the contraction of the deep flexors in different positions in the following sessions (Figure 1). In sessions 2, 3, and 4 with the therapist, exercises involving other muscles and different movements (flexion/extension/rotations/inclinations) were implemented. External resistance was used to increase the intensity of the exercises and was advanced unilaterally towards the most symptomatic side (Figure 2). All exercises were performed with prior contraction of the deep flexors [36]. Moreover, all patients were encouraged to perform home exercises every day between two and five times a day, starting after the first session [37,39-41].
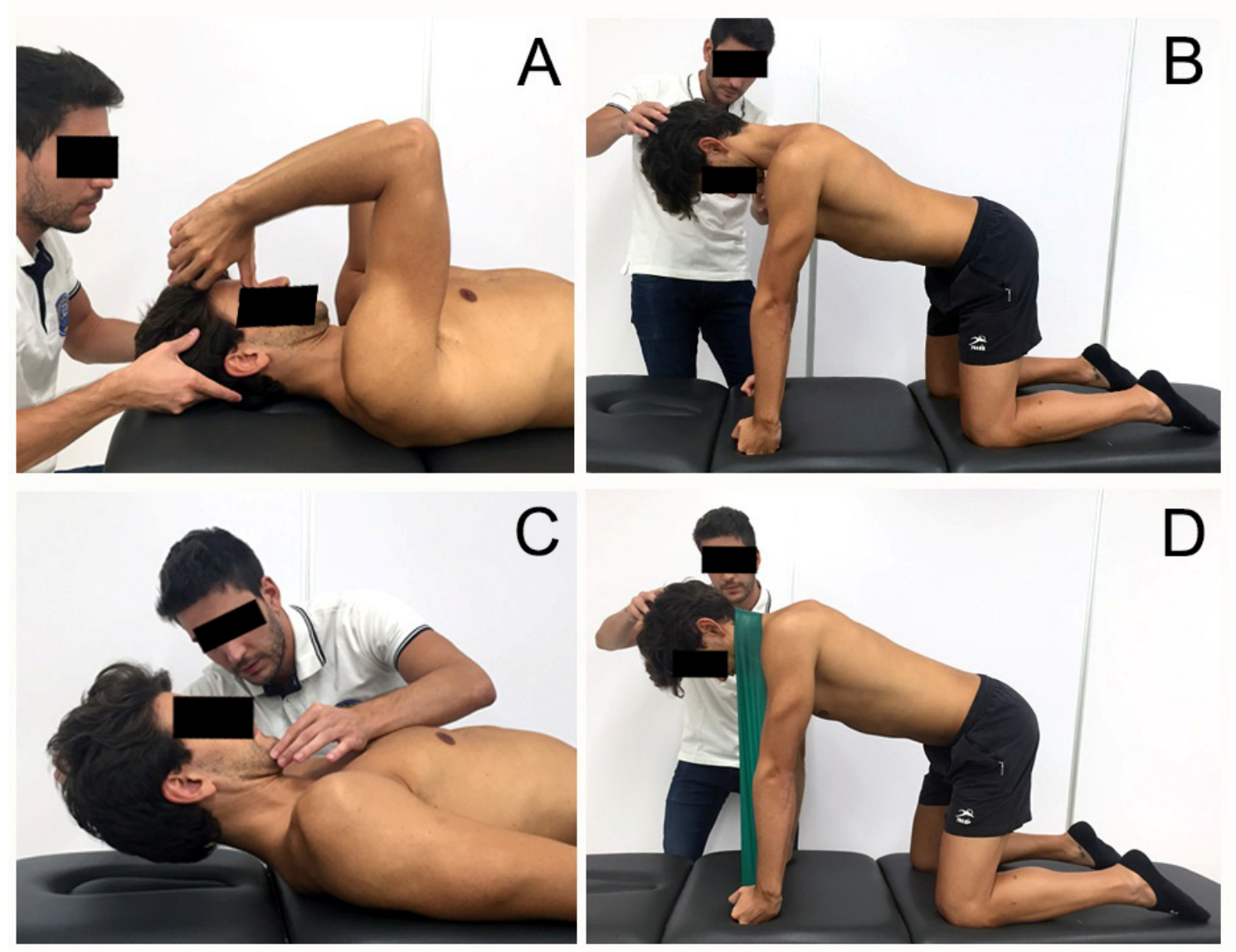

Figure 2. Progression exercises involving superficial muscles. (A) Bilateral isometric contraction of superficial and deep flexors muscles; (B) Bilateral contraction of deep flexors and extensors in four points; (C) Bilateral isometric contraction of superficial and deep flexors muscles against gravity; (D) An example of Bilateral contraction of deep flexors and extensors in quadruped with resistance.

\subsection{Manual Therapy + Exercise Group $(M T+E)$}

The MT + E program was conducted one day a week for four weeks with the same duration as the Exercise group. Manipulation (high velocity, low amplitude) (Figure 3) and/or mobilization (low velocity, high amplitude) techniques (Figure 4) of the upper cervical spine were combined with cervical exercise $[8,17,23,42,43]$. The manual therapy techniques used depended on each patient's clinical findings. The manual therapy approach aimed to restore the mobility of the upper cervical joints by treating occipital-atlas (C0-1) and axis-C3 (C2-3) and then, if necessary, atlas-axis (C1-2) segment. The manipulation and mobilization techniques were always performed with translatoric movements. All the techniques followed the International Federation of Orthopaedic Manipulative 
Physical Therapists (IFOMPT) recommendations to reduce the risk of adverse effects [19]. The training exercises followed the same progression as the exercise group. The MT + E group rested 30-s between repetitions instead of 40-s during the exercise. This was done to have time to apply the manual therapy techniques and maintain the same session length as the exercise group (20 $\mathrm{min})$.

All patients were encouraged to perform the self-treatment exercises at home every day between two and five times a day after the first session [37,39-41].
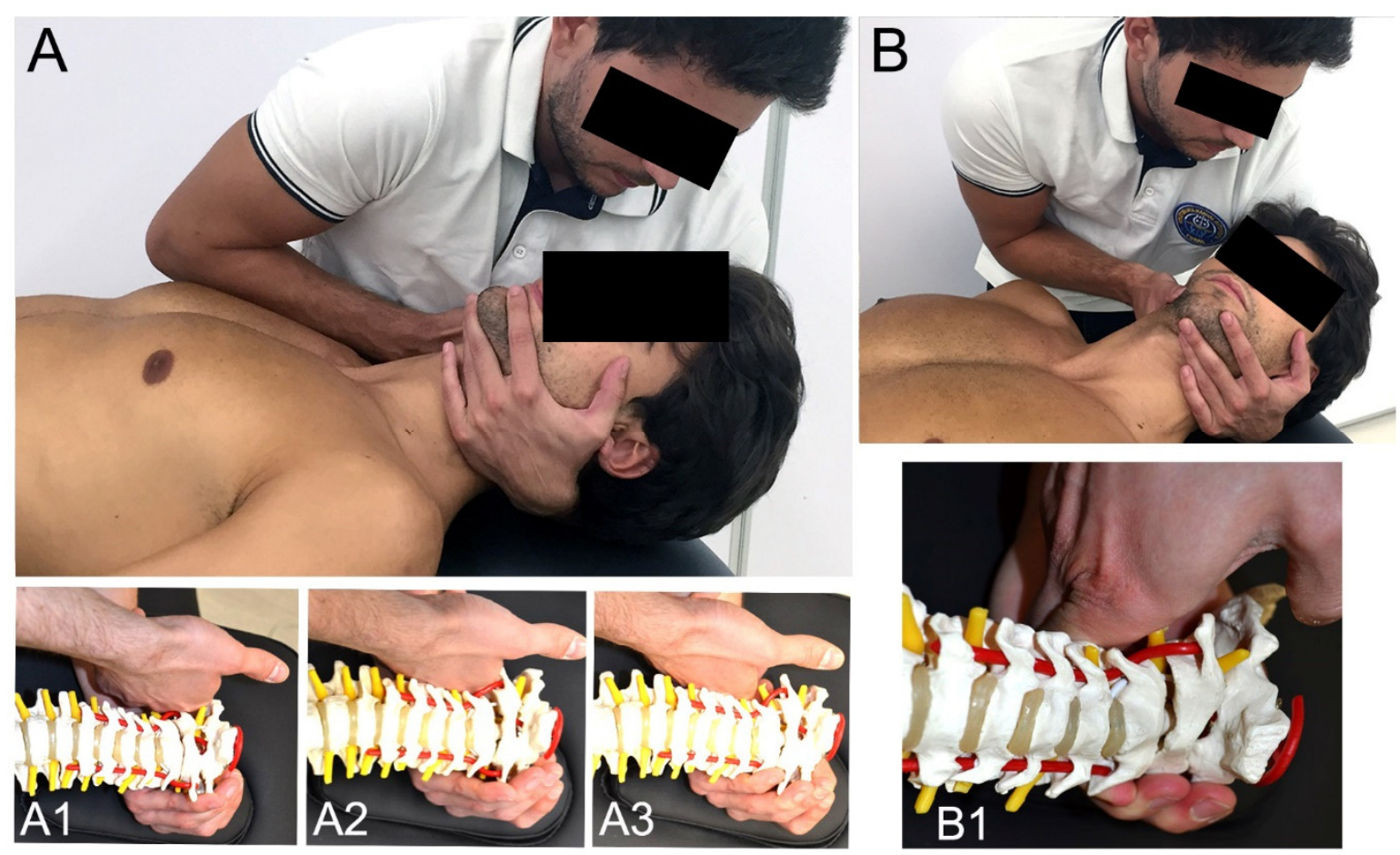

Figure 3. Manipulation Techniques. (A) Traction-Manipulation in the Resting Position; (A1) TractionManipulation in the Resting Position C0-1; (A2) Traction-Manipulation in the Resting Position C1-2; (A3) Traction-Manipulation in the Resting Position C2-3; (B,B1) Facet Traction-Manipulation C2-3.
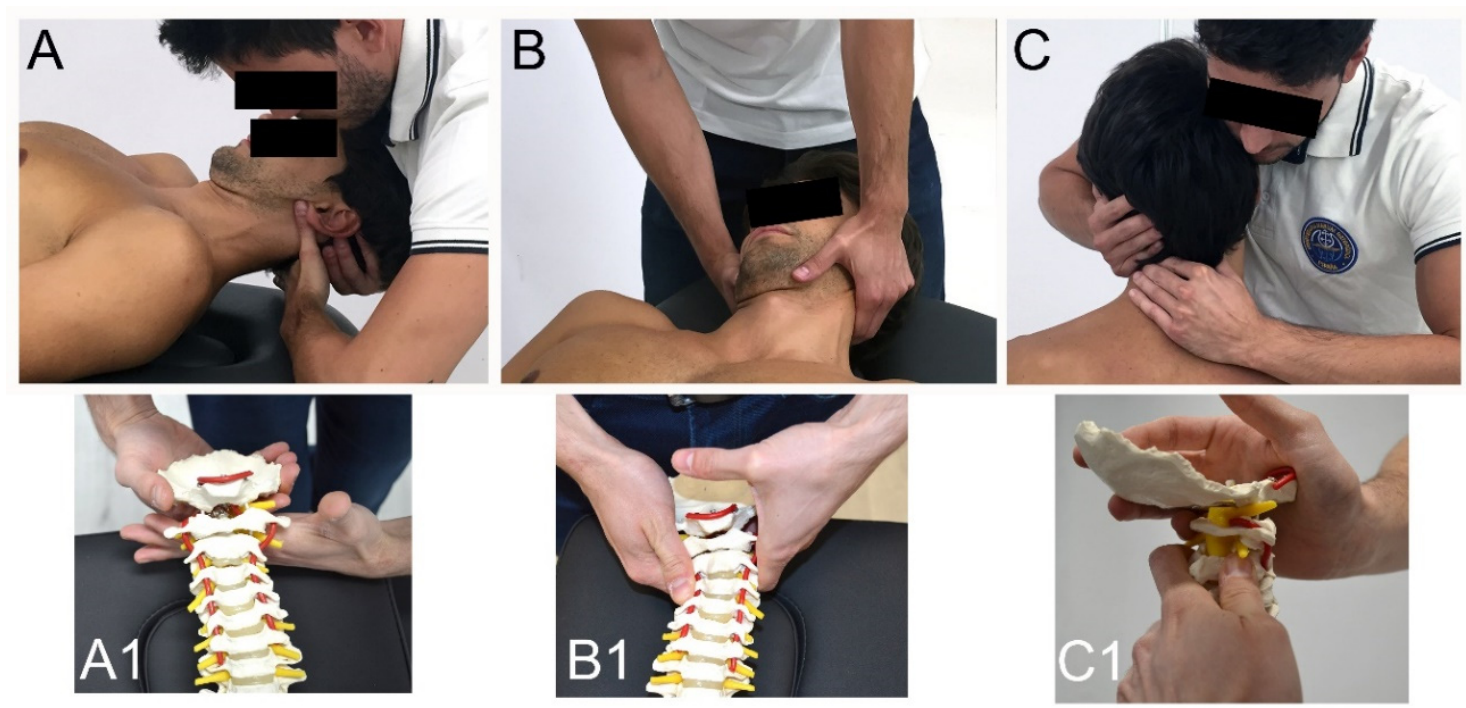

Figure 4. Mobilization Techniques. (A,A1) Upper Cervical Translatoric Dorsal Glide C0-1; (B,B1) VentralCranial Glide C2-3 in supine (C,C1) Upper Cervical Translatoric Dorsal Glide C1-2. 


\subsection{Statistical Analysis}

Statistical analysis was conducted using SPSS 25.0 package (IBM, Armonk, NY, USA). The mean and standard deviations were calculated for each variable. The Kolmogorov-Smirnov test was used to determine a normal distribution of quantitative data $(p>0.05)$. Within- and between-group differences were analyzed using repeated-measures ANOVA and one-way ANOVA for quantitative variables. For qualitative variables, Fisher's exact test was used. Effect sizes were calculated using Cohen's d coefficient [44]. An effect size $>0.8$ was considered large; around 0.5, intermediate; and $<0.2$, small [44]. Losses and exclusions after randomization are explained in Figure 5. The statistical analysis was performed on an intention-to-treat basis. The level of significance was set at $p<0.05$.

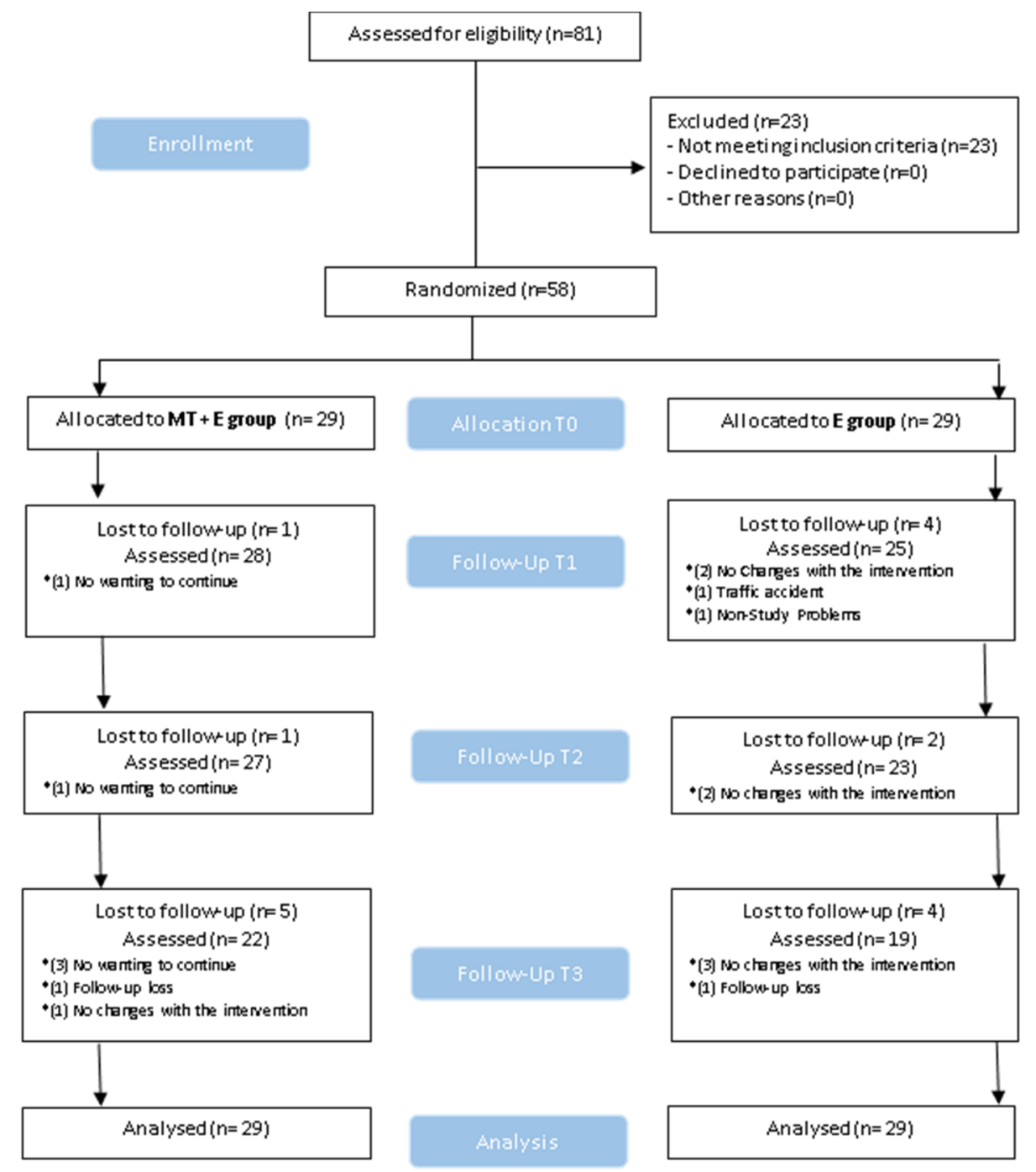

Figure 5. CONSORT. (Consolidated Standards of Reporting Trial) flow diagram.

\section{Results}

Between October 2018 and January 2020, eighty-one volunteers were recruited. Fifty-eight participants (17 men, 41 women) with a mean age of 49.2 (15.9) met all eligibility criteria and agreed to participate. Then 29 participants were randomly assigned to each group, received their assigned treatment, and were analyzed for intention to treat (Figure 5). The demographic characteristics of the sample are summarized in Table 1. Drop-outs, enrollment, exclusions after randomization, and follow-ups are in the flow diagram (Figure 5). There were no adverse events with the treatments 
performed in the study at any follow-up. However, three exercise group participants reported treatment side-effects, such as mild and transient aggravation of neck pain, in the 6-month follow-up.

Table 1. Baseline features for both groups.

\begin{tabular}{|c|c|c|}
\hline Clinical Features & E Group $(n=29)$ & MT + E Group $(n=29)$ \\
\hline Age (years) & $49.72 \pm 17.56$ & $48.76 \pm 14.53$ \\
\hline Sex & $7 \mathrm{M} ; 22 \mathrm{~F}$ & $10 \mathrm{M} ; 19 \mathrm{~F}$ \\
\hline Duration of Symptoms (months) & $124.38 \pm 148.17$ & $96.97 \pm 96.73$ \\
\hline $\begin{array}{l}\text { Visual Analogue Scale } \\
\text { (centimeters) }\end{array}$ & $3.76 \pm 2.53$ & $3.36 \pm 1.97$ \\
\hline Upper Cervical Flexion $\left(^{\circ}\right)$ & $10.59 \pm 4.39$ & $11.45 \pm 4.24$ \\
\hline Neck Disability Index & $15.24 \pm 6.99$ & $12.55 \pm 6.25$ \\
\hline Doesn't get it & $51.70 \%$ & $34.50 \%$ \\
\hline $20 \mathrm{mmHg}$ & $17.20 \%$ & $34.50 \%$ \\
\hline $22 \mathrm{mmHg}$ & $31 \%$ & $31 \%$ \\
\hline \multicolumn{3}{|l|}{ Flexion-rotation test $\left(^{\circ}\right)$} \\
\hline$(+)$ & $12.80 \pm 6.04$ & $17.26 \pm 7.90$ \\
\hline$(-)$ & $22.91 \pm 10.52$ & $27.12 \pm 9.19$ \\
\hline \multicolumn{3}{|l|}{ Pressure Pain Threshold (kPa) } \\
\hline First MCJ $(\mathrm{R})$ & $359.14 \pm 175.98$ & $395.93 \pm 195.23$ \\
\hline $\mathrm{C} 2-3(\mathrm{R})$ & $173.76 \pm 87.92$ & $208.69 \pm 114.53$ \\
\hline Suboccipital (R) & $186.10 \pm 75.34$ & $211.45 \pm 91.57$ \\
\hline First MCJ (L) & $364.34 \pm 155.47$ & $339.90 \pm 184.74$ \\
\hline $\mathrm{C} 2-3(\mathrm{~L})$ & $174.59 \pm 90.02$ & $206.38 \pm 113.72$ \\
\hline Suboccipital (L) & $180.59 \pm 79.85$ & $207.90 \pm 105.33$ \\
\hline
\end{tabular}

$\mathrm{M}$, male; $\overline{\mathrm{F}}$, female; Doesn't get it, the patient is unable to start the test because of pain; $20-22 \mathrm{mmHg}$, the patient is able to complete the test up to the indicated millimeters of mercury + , most restricted or less range of movement between the two test rotations; -, less restricted or higher range of movement between the two test rotations; ROM, range of motion; R, right; L, left; MCJ, metacarpal joint; E, Exercise; MT + E, Manual Therapy + Exercise.

\subsection{End of the Intervention (T1)}

In the within-group analysis of the exercise group, statistically significant improvement was found in the neck disability index questionnaire and the craniocervical flexion test $(p<0.01)$ (Figure 6). In the $\mathrm{MT}+\mathrm{E}$, significant improvement was found in the following variables: visual analogue scale, neck disability index, flexion-rotation test to the more restricted $(+)$ and less restricted $(-)$ side $(p<0.01)$ (Table 2), pressure pain threshold in the suboccipital (right), C2-3 (left), and suboccipital (left) $(p<0.05)$ and craniocervical flexion test (Figure 6). 
Table 2. Outcomes variable values within-group.

\begin{tabular}{|c|c|c|c|c|c|c|c|c|c|c|c|c|c|c|c|c|c|}
\hline \multirow{3}{*}{ Group } & \multirow{3}{*}{ Variable } & \multirow{3}{*}{$\begin{array}{c}\text { T0 } \\
\text { Baseline } \\
\text { Mean } \pm \text { SD } \\
\end{array}$} & \multicolumn{5}{|c|}{ T1 } & \multicolumn{5}{|c|}{ T2 } & \multicolumn{5}{|c|}{ T3 } \\
\hline & & & \multirow{2}{*}{$\begin{array}{c}1 \text { Month } \\
\text { Mean } \pm \text { SD }\end{array}$} & \multicolumn{3}{|c|}{ Difference between Baseline } & \multicolumn{2}{|r|}{3 Months } & \multicolumn{3}{|c|}{ Difference between Baseline } & \multicolumn{2}{|r|}{6 Months } & \multicolumn{3}{|c|}{ Difference between Baseline } & \multirow[b]{2}{*}{$\mathrm{d}$} \\
\hline & & & & Mean \pm SD & $F$ & $p$-Value ${ }^{R A}$ & $\mathrm{~d}$ & Mean \pm SD & Mean \pm SD & $F$ & $p$-Value ${ }^{R A}$ & $\mathrm{~d}$ & Mean \pm SD & Mean \pm SD & $\mathrm{F}$ & $p$-Value ${ }^{R A}$ & \\
\hline \multirow{12}{*}{ 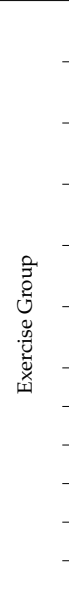 } & $\begin{array}{c}\text { Visual Analogue } \\
\text { Scale (centimeters) }\end{array}$ & $3.76 \pm 2.53$ & $2.89 \pm 2.44$ & $-0.87 \pm 0.09$ & 2.89 & $>0.661$ & 0.35 & $3.87 \pm 2.71$ & $0.11 \pm 0.18$ & 2.89 & $>1.000$ & 0.04 & $3.91 \pm 2.84$ & $0.15 \pm 0.31$ & 2.89 & $>1.000$ & 0.06 \\
\hline & $\begin{array}{l}\text { Upper Cervical } \\
\text { Flexion }\left(^{\circ}\right)\end{array}$ & $10.59 \pm 4.39$ & $10.59 \pm 5.21$ & $0.00 \pm 0.82$ & 2.57 & $>1.000$ & 0.00 & $9.03 \pm 5.27$ & $-1.56 \pm 0.88$ & 2.57 & $>0.406$ & 0.32 & $8.93 \pm 4.76$ & $-1.66 \pm 0.37$ & 2.57 & $>0.177$ & 0.36 \\
\hline & $\begin{array}{l}\text { Neck Disability } \\
\text { Index }\end{array}$ & $15.24 \pm 6.99$ & $11.03 \pm 6.74$ & $-4.21 \pm 0.25$ & 6.39 & $<0.001$ & 0.61 & $12.83 \pm 8.09$ & $-2.41 \pm 1.10$ & 6.39 & $>0.116$ & 0.32 & $13.10 \pm 8.58$ & $-2.14 \pm 1.59$ & 6.39 & $>0.420$ & 0.27 \\
\hline & $\begin{array}{l}\text { Flexion-rotation test } \\
+\left(\left(^{\circ}\right)\right.\end{array}$ & $12.80 \pm 6.04$ & $15.48 \pm 10.34$ & $2.68 \pm 4.30$ & 2.27 & $>0.489$ & 0.32 & $12.76 \pm 7.77$ & $-0.04 \pm 1.73$ & 2.27 & $>1.000$ & 0.01 & $12.83 \pm 9.10$ & $0.03 \pm 3.06$ & 2.27 & $>1.000$ & 0.00 \\
\hline & $\begin{array}{l}\text { Flexion-rotation } \\
\text { test- }\left({ }^{\circ}\right)\end{array}$ & $22.91 \pm 10.52$ & $23.59 \pm 11.21$ & $0.68 \pm 0.69$ & 8.52 & $>1.000$ & 0.06 & $17.21 \pm 8.54$ & $-5.70 \pm 1.98$ & 8.52 & $<0.002$ & 0.60 & $18.03 \pm 10.42$ & $-4.88 \pm 0.10$ & 8.52 & $<0.024$ & 0.47 \\
\hline & $\begin{array}{l}\text { Pressure Pain } \\
\text { Threshold (kpa) }\end{array}$ & & & & & & & & & & & & & & & & \\
\hline & First MCJ (R) & $359.14 \pm 175.98$ & $351.24 \pm 168.84$ & $-7.90 \pm 7.14$ & 2.24 & $>1.000$ & 0.05 & $310.21 \pm 133.37$ & $-48.93 \pm 42.61$ & 2.24 & $>0.145$ & 0.31 & $304.66 \pm 121.12$ & $-54.48 \pm 54.86$ & 2.24 & $>0.198$ & 0.36 \\
\hline & $\mathrm{C} 2-3(\mathrm{R})$ & $173.76 \pm 87.92$ & $166.48 \pm 78.91$ & $-7.28 \pm 9.01$ & 2.06 & $>1.000$ & 0.09 & $149.76 \pm 77.08$ & $-24.00 \pm 10.84$ & 2.06 & $>0.542$ & 0.29 & $145.93 \pm 65.41$ & $-27.83 \pm 22.51$ & 2.06 & $>0.258$ & 0.36 \\
\hline & Suboccipital (R) & $186.10 \pm 75.34$ & $180.79 \pm 81.65$ & $-5.31 \pm 6.31$ & 0.97 & $>1.000$ & 0.07 & $161.59 \pm 79.99$ & $-24.51 \pm 4.65$ & 0.97 & $>0.722$ & 0.32 & $163.97 \pm 76.99$ & $-22.13 \pm 1.65$ & 0.97 & $>0.943$ & 0.29 \\
\hline & First MCJ (L) & $364.34 \pm 155.47$ & $343.45 \pm 177.71$ & $-20.89 \pm 22.24$ & 1.35 & $>1.000$ & 0.13 & $339.93 \pm 171.41$ & $-24.41 \pm 15.94$ & 1.35 & $>1.000$ & 0.15 & $323.41 \pm 148.53$ & $-40.93 \pm 6.94$ & 1.35 & $>0.289$ & 0.27 \\
\hline & $\mathrm{C} 2-3(\mathrm{~L})$ & $174.59 \pm 90.02$ & $185.90 \pm 80.34$ & $11.31 \pm 9.68$ & 1.91 & $>1.000$ & 0.13 & $159.97 \pm 80.15$ & $-14.62 \pm 9.87$ & 1.91 & $>1.000$ & 0.17 & $168.76 \pm 76.65$ & $-5.83 \pm 13.37$ & 1.91 & $>1.000$ & 0.07 \\
\hline & Suboccipital (L) & $180.59 \pm 79.85$ & $196.90 \pm 79.97$ & $16.31 \pm 0.08$ & 0.61 & $>1.000$ & 0.20 & $181.90 \pm 67.35$ & $1.31 \pm 12.50$ & 0.61 & $>1.000$ & 0.02 & $182.45 \pm 65.61$ & $1.86 \pm 14.24$ & 0.61 & $>1.000$ & 0.03 \\
\hline
\end{tabular}


Table 2. Cont.

\begin{tabular}{|c|c|c|c|c|c|c|c|c|c|c|c|c|c|c|c|c|c|}
\hline \multirow{3}{*}{ Group } & \multirow{3}{*}{ Variable } & \multirow{3}{*}{$\begin{array}{c}\text { T0 } \\
\text { Baseline } \\
\text { Mean } \pm \text { SD } \\
\end{array}$} & \multicolumn{5}{|c|}{ T1 } & \multicolumn{5}{|c|}{ T2 } & \multicolumn{5}{|c|}{$\mathrm{T} 3$} \\
\hline & & & \multirow{2}{*}{$\begin{array}{c}\text { 1 Month } \\
\text { Mean } \pm \text { SD }\end{array}$} & \multicolumn{3}{|c|}{ Difference between Baseline } & \multirow[b]{2}{*}{$\mathrm{d}$} & \multirow{2}{*}{\begin{tabular}{|c|}
3 Months \\
Mean \pm SD
\end{tabular}} & \multicolumn{3}{|c|}{ Difference between Baseline } & \multicolumn{2}{|r|}{6 Months } & \multicolumn{4}{|c|}{ Difference between Baseline } \\
\hline & & & & Mean \pm SD & F & $p$-Value ${ }^{R A}$ & & & Mean \pm SD & F & $p$-Value ${ }^{R A}$ & $\mathrm{~d}$ & Mean \pm SD & Mean \pm SD & F & $p$-Value ${ }^{R A}$ & $\mathrm{~d}$ \\
\hline \multirow{12}{*}{ 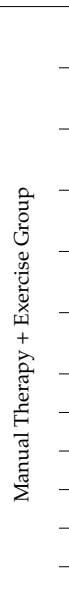 } & $\begin{array}{c}\text { Visual Analogue } \\
\text { Scale (centimeters) }\end{array}$ & $3.36 \pm 1.97$ & $0.75 \pm 1.42$ & $-2.61 \pm 0.55$ & 13.91 & $<0.001$ & 1.52 & $0.80 \pm 1.30$ & $-2.56 \pm 1.42$ & 13.91 & $<0.001$ & 1.53 & $0.98 \pm 1.49$ & $-2.38 \pm 0.48$ & 13.91 & $1<0.001$ & 1.36 \\
\hline & $\begin{array}{l}\text { Upper Cervical } \\
\text { Flexion }\left({ }^{\circ}\right)\end{array}$ & $11.45 \pm 4.24$ & $13.55 \pm 4.13$ & $2.10 \pm 0.11$ & 10.84 & $>0.074$ & 0.50 & $14.83 \pm 4.63$ & $3.38 \pm 0.39$ & 10.84 & $<0.022$ & 0.76 & $16.90 \pm 4.83$ & $5.45 \pm 0.59$ & 10.84 & $4<0.001$ & 1.20 \\
\hline & $\begin{array}{l}\text { Neck Disability } \\
\text { Index }\end{array}$ & $12.55 \pm 6.25$ & $5.45 \pm 5.53$ & $-7.10 \pm 0.72$ & 19.26 & $<0.001$ & 1.20 & $4.66 \pm 5.62$ & $-7.89 \pm 0.63$ & 19.26 & $<0.001$ & 1.33 & $4.76 \pm 5.96$ & $-7.79 \pm 0.29$ & 19.26 & $6<0.001$ & 1.28 \\
\hline & $\begin{array}{c}\text { Flexion-rotation test } \\
+\left({ }^{\circ}\right)\end{array}$ & $17.26 \pm 7.90$ & $37.79 \pm 10.48$ & $20.53 \pm 2.58$ & 42.51 & $<0.001$ & 2.21 & $35.83 \pm 9.61$ & $18.57 \pm 1.71$ & 42.51 & $<0.001$ & 2.25 & $34.48 \pm 12.04$ & $17.22 \pm 4.14$ & 42.51 & $1<0.001$ & 1.69 \\
\hline & $\begin{array}{l}\text { Flexion-rotation } \\
\text { test- }\left({ }^{\circ}\right)\end{array}$ & $27.12 \pm 9.19$ & $41.97 \pm 9.47$ & $14.85 \pm 0.28$ & 31.50 & $<0.001$ & 1.59 & $40.07 \pm 8.16$ & $12.95 \pm 1.03$ & 31.50 & $<0.001$ & 1.49 & $38.66 \pm 9.42$ & $11.54 \pm 0.23$ & 31.50 & $0<0.001$ & 1.24 \\
\hline & $\begin{array}{l}\text { Pressure Pain } \\
\text { Threshold (Kpa) }\end{array}$ & & & & & & & & & & & & & & & & \\
\hline & First MCJ (R) & $395.93 \pm 195.23$ & $417.14 \pm 194.94$ & $21.21 \pm 0.29$ & 3.72 & $>1.000$ & 0.11 & $431.00 \pm 193.54$ & $35.07 \pm 1.69$ & 3.72 & $>1.000$ & 0.18 & $479.00 \pm 214.95$ & $83.07 \pm 19.72$ & 3.72 & $>0.182$ & 0.41 \\
\hline & $\mathrm{C} 2-3(\mathrm{R})$ & $208.69 \pm 114.53$ & $250.83 \pm 113.26$ & $42.14 \pm 1.27$ & 4.56 & $>0.108$ & 0.33 & $277.86 \pm 135.35$ & $19.17 \pm 20.82$ & 4.56 & $<0.016$ & 0.49 & $305.83 \pm 162.70$ & $97.14 \pm 48.17$ & 4.56 & $<0.004$ & 0.69 \\
\hline & Suboccipital (R) & $211.45 \pm 91.57$ & $257.55 \pm 112.31$ & $46.10 \pm 20.74$ & 10.35 & $<0.016$ & 0.45 & $297.72 \pm 117.74$ & $86.27 \pm 26.17$ & 10.35 & $<0.001$ & 0.82 & $344.48 \pm 171.01$ & $133.03 \pm 79.44$ & 10.35 & $5<0.001$ & 0.97 \\
\hline & First MCJ (L) & $339.90 \pm 184.74$ & $397.97 \pm 172.87$ & $58.07 \pm 11.87$ & 6.12 & $>0.387$ & 0.33 & $421.72 \pm 178.22$ & $81.82 \pm 6.52$ & 6.12 & $>0.120$ & 0.45 & $483.52 \pm 198.00$ & $143.62 \pm 13.26$ & 6.12 & $<0.003$ & 0.75 \\
\hline & $\mathrm{C} 2-3(\mathrm{~L})$ & $206.38 \pm 113.72$ & $279.76 \pm 167.66$ & $73.38 \pm 53.94$ & 10.19 & $<0.004$ & 0.51 & $298.14 \pm 161.58$ & $91.76 \pm 47.86$ & 10.19 & $<0.001$ & 0.66 & $332.52 \pm 166.91$ & $126.14 \pm 53.19$ & 10.19 & $9<0.001$ & 0.88 \\
\hline & Suboccipital (L) & $207.90 \pm 105.33$ & $267.00 \pm 116.26$ & $59.10 \pm 10.93$ & 15.22 & $<0.002$ & 0.53 & $314.14 \pm 155.73$ & $106.24 \pm 50.40$ & 15.22 & $<0.001$ & 0.80 & $380.66 \pm 198.09$ & $172.76 \pm 92.76$ & 15.22 & $2<0.001$ & 1.09 \\
\hline
\end{tabular}

Abreviature: SD, Standard Deviation; +, most restricted or less range of movement between the two test rotations; - less restricted or higher range of movement between the two test rotations; MCJ, First Metacarpal Joint; R, Right; L, Left; ${ }^{R A}$. Repeated measures ANOVA; d. Cohen's d coefficient. 


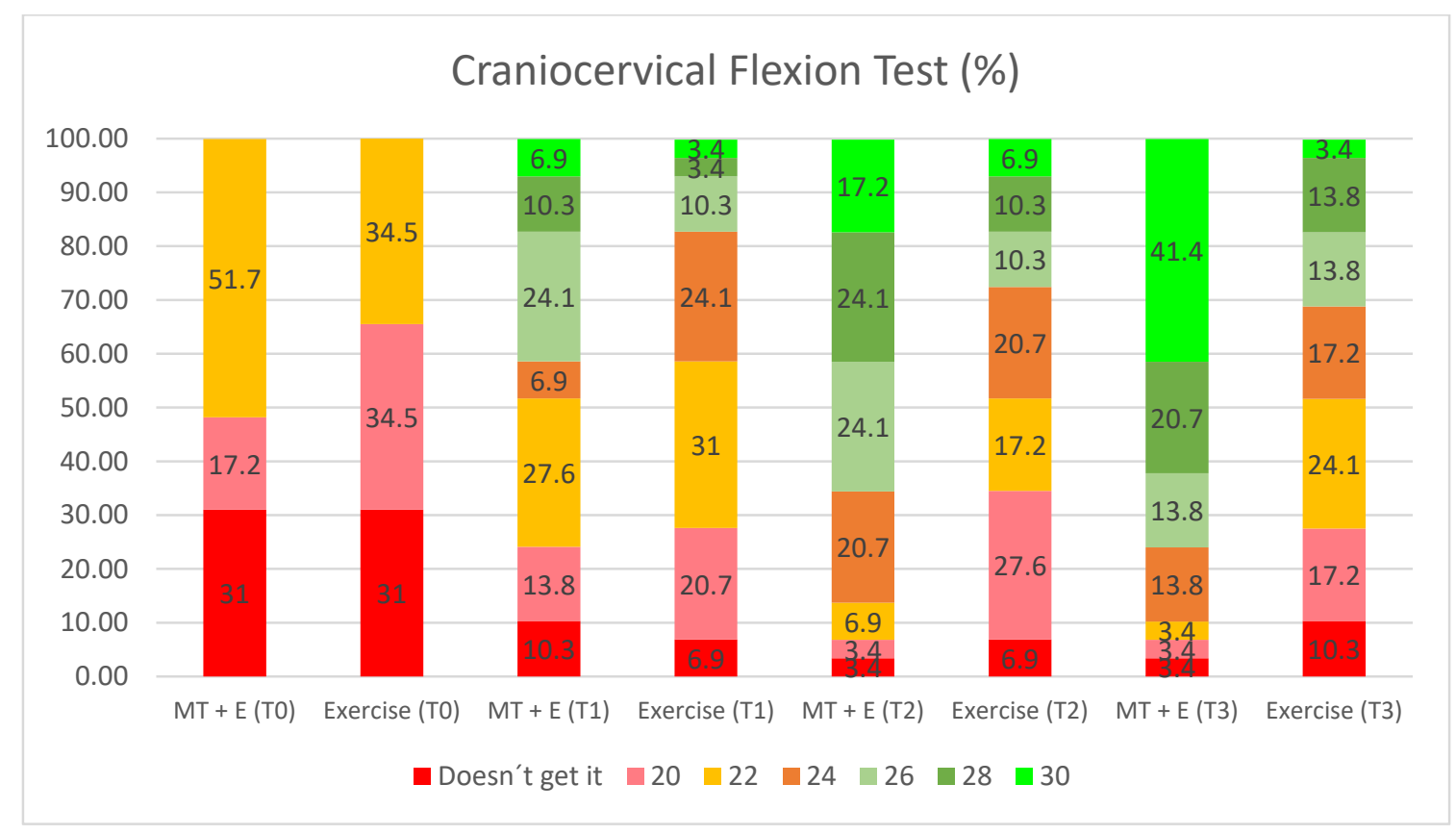

Figure 6. Craniocervical Flexion Test Graphic. Doesn't get it, the patient is unable to start the test because of pain; $20-30 \mathrm{mmHg}$, the patient is able to complete the test up to the indicated millimeters of mercury MT + E, Manual Therapy + Exercise.

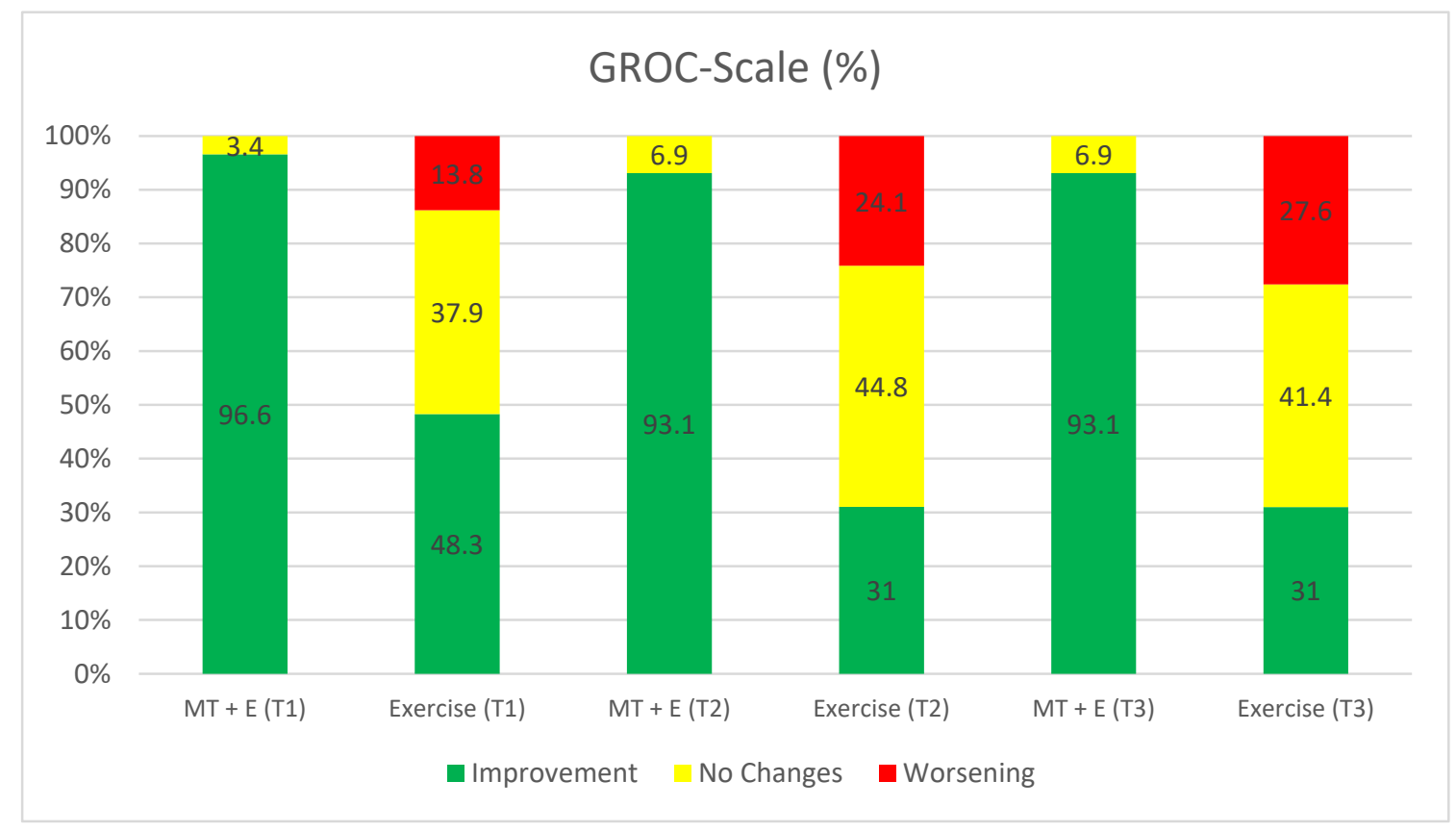

Figure 7. Global rating of change scale (GROC-Scale) Graphic. Patients' subjective perceptions of clinical change. MT + E, Manual Therapy + Exercise.

In the between-group analysis (Table 3), statistically significant differences were found in favor of the MT + E group in the visual analogue scale, neck disability index, flexion-rotation test to the more restricted $(+)$ and less restricted $(-)$ side $(p<0.05)$ and in the pressure pain threshold variables in C2-3 (right), suboccipital (right), first metacarpal joint (left), C2-3 (left), and suboccipital (left) $(p<0.05)$. Differences in the GROC-Scale variable $(p<0.01)$ (Figure 7) were also identified. 
Table 3. Outcomes variable values between-group.

\begin{tabular}{|c|c|c|c|c|c|c|c|c|c|c|c|c|c|c|c|c|c|}
\hline \multirow{3}{*}{ Variable } & \multirow{3}{*}{ Group } & \multirow{3}{*}{$\begin{array}{c}\text { T0 } \\
\text { Baseline } \\
\text { Mean } \pm \text { SD }\end{array}$} & \multicolumn{5}{|c|}{ T1 } & \multicolumn{5}{|c|}{ T2 } & \multicolumn{5}{|c|}{ T3 } \\
\hline & & & \multirow{2}{*}{$\begin{array}{c}\text { 1 Month } \\
\text { Mean } \pm \text { SD }\end{array}$} & \multicolumn{4}{|c|}{ Difference between Groups } & 3 Months & \multicolumn{3}{|c|}{ Difference between Groups } & \multicolumn{2}{|r|}{6 Months } & \multicolumn{3}{|c|}{ Difference between Groups } & \multirow[b]{2}{*}{ d } \\
\hline & & & & Mean \pm SD & $\mathrm{F}$ & $p$-Value ${ }^{O A}$ & d & Mean \pm SD & Mean \pm SD & $\mathrm{F}$ & $p$-Value ${ }^{O A}$ & d & Mean $\pm \mathrm{SD}$ & Mean \pm SD & $\mathrm{F}$ & $p$-Value ${ }^{O A}$ & \\
\hline \multirow{2}{*}{$\begin{array}{l}\text { Visual Analogue } \\
\text { Scale } \\
\text { (centimeters) }\end{array}$} & E Group & $3.76 \pm 2.53$ & $2.89 \pm 2.44$ & \multirow{2}{*}{$2.14 \pm 1.02$} & \multirow{2}{*}{6.23} & \multirow{2}{*}{$3<0.016$} & \multirow{2}{*}{0.78} & $3.87 \pm 2.71$ & \multirow{2}{*}{$3.07 \pm 1.41$} & \multirow{2}{*}{15.50} & \multirow{2}{*}{$<0.001$} & \multirow{2}{*}{1.44} & $3.91 \pm 2.84$ & \multirow{2}{*}{$2.93 \pm 1.35$} & \multirow{2}{*}{14.73} & \multirow{2}{*}{$3<0.001$} & \multirow{2}{*}{1.29} \\
\hline & MT + E Group & $3.36 \pm 1.97$ & $0.75 \pm 1.42$ & & & & & $0.80 \pm 1.30$ & & & & & $0.98 \pm 1.49$ & & & & \\
\hline \multirow{2}{*}{$\begin{array}{l}\text { Upper Cervical } \\
\text { Flexion }\left(^{\circ}\right)\end{array}$} & E Group & $10.59 \pm 4.39$ & $10.59 \pm 5.21$ & \multirow{2}{*}{$2.96 \pm 1.08$} & 271 & $1>0078$ & 063 & $9.03 \pm 5.27$ & $580+061$ & 1217 & -0001 & 1.17 & $8.93 \pm 4.76$ & $707+007$ & 36.33 & $33-0001$ & 16 \\
\hline & MT + E Group & $11.45 \pm 4.24$ & $13.55 \pm 4.13$ & & 3.14 & $=>0.058$ & 0.63 & $14.83 \pm 4.63$ & $5.80 \pm 0.64$ & 13.47 & $<0.001$ & & $16.90 \pm 4.83$ & . & 36.33 & $83<0.001$ & 1.66 \\
\hline Neck Disability & E Group & $15.24 \pm 6.99$ & $11.03 \pm 6.74$ & $558+121$ & 4.35 & $5<0042$ & 074 & $12.83 \pm 8.09$ & $817+247$ & 1478 & $<0001$ & 117 & $13.10 \pm 8.58$ & $834+262$ & 1221 & $1<0001$ & 113 \\
\hline Index & MT + E Group & $12.55 \pm 6.25$ & $5.45 \pm 5.53$ & $.30 \pm \pm 1.21$ & 4.30 & $p<0.042$ & 0.14 & $4.66 \pm 5.62$ & $0.17 \pm 2.4 /$ & 14.18 & $<0.001$ & 1.17 & $4.76 \pm 5.96$ & $8.34 \pm 2.02$ & 12.21 & $1.1<0.001$ & 1.13 \\
\hline Flexion-rotation & E Group & $12.80 \pm 6.04$ & $15.48 \pm 10.34$ & & & $17<0001$ & & $12.76 \pm 7.77$ & & & & & $12.83 \pm 9.10$ & $21,65+294$ & & & \\
\hline test $+\left(^{\circ}\right)$ & MT + E Group & $17.26 \pm 7.90$ & $37.79 \pm 10.48$ & $22.31 \pm 0.14$ & 53.47 & 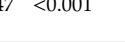 & 2.14 & $35.83 \pm 9.61$ & $23.07 \pm 1.84$ & 78.84 & $<0.001$ & 2.64 & $34.48 \pm 12.04$ & $21.65 \pm 2.94$ & 45.55 & $5<0.001$ & 2.03 \\
\hline Flexion-rotation & E Group & $22.91 \pm 10.52$ & $23.59 \pm 11.21$ & & & & & $17.21 \pm 8.54$ & & & & & $18.03 \pm 10.42$ & & & & \\
\hline test- $\left({ }^{\circ}\right)$ & MT + E Group & $27.12 \pm 9.19$ & $41.97 \pm 9.47$ & $18.38 \pm 1.74$ & 44.37 & $37<0.001$ & 1.77 & $40.07 \pm 8.16$ & $22.86 \pm 0.38$ & 70.91 & $<0.001$ & 2.74 & $38.66 \pm 9.42$ & $20.63 \pm 1.00$ & 59.36 & $36<0.001$ & 2.08 \\
\hline $\begin{array}{l}\text { Pressure Pain } \\
\text { Threshold (Kpa) }\end{array}$ & & & & & & & & & & & & & & & & & \\
\hline First MCI (R) & E Group & $359.14 \pm 175.98$ & $351.24 \pm 168.84$ & & & & & $310.21 \pm 133.37$ & & & & & $304.66 \pm 121.12$ & & & & \\
\hline & MT + E Group & $395.93 \pm 195.23$ & $417.14 \pm 194.94$ & $65.90 \pm 26.10$ & 0.53 & $3>0.471$ & 0.36 & $431.00 \pm 193.54$ & $120.79 \pm 60.17$ & 4.14 & $<0.047$ & 0.73 & $479.00 \pm 214.95$ & $174.34 \pm 93.83$ & 9.88 & $3<0.003$ & 1.00 \\
\hline$C_{2}-3(\mathrm{R})$ & E Group & $173.76 \pm 87.92$ & $166.48 \pm 78.91$ & $8425+3432$ & 571 & 1 & 086 & $149.76 \pm 77.08$ & $17810+577$ & 1280 & 50001 & 116 & $145.93 \pm 65.41$ & $15090+07$ ? & 1001 & 50001 & 120 \\
\hline$C 2-3$ (I) & MT + E Group & $208.69 \pm 114.53$ & $250.83 \pm 113.26$ & $84.35 \pm 34.35$ & $5 . / 4$ & $t<<.020$ & 0.86 & $277.86 \pm 135.35$ & $128.10 \pm 58.27$ & 13.89 & $<0.001$ & 1.16 & $305.83 \pm 162.70$ & $159.90 \pm 97.29$ & 19.01 & $11<0.001$ & 1.29 \\
\hline Suboccipital (R) & E Group & $186.10 \pm 75.34$ & $180.79 \pm 81.65$ & $7676+3066$ & 735 & $<0009$ & 078 & $161.59 \pm 79.99$ & $13613+3715$ & 2537 & $<0001$ & 135 & $163.97 \pm 76.99$ & $180.51+94.02$ & $28,23 \quad \longrightarrow$ & $03<0001$ & $136 \mathrm{r}-\mathrm{s}$ \\
\hline & MT + E Group & $211.45 \pm 91.57$ & $257.55 \pm 112.31$ & $70.16 \pm 30.60$ & 7.30 & $8<0.009$ & 0.18 & $297.72 \pm 117.74$ & $100.10 \pm 3 / .13$ & 20.37 & $<0.001$ & 1.30 & $344.48 \pm 171.01$ & $180.51 \pm 94.02$ & 28.23 & $3<<0.001$ & 1.06 \\
\hline First MCI (L) & E Group & $364.34 \pm 155.47$ & $343.45 \pm 177.71$ & $5452+484$ & 410 & $\leq 0048$ & 031 & $339.93 \pm 171.41$ & $8179+681$ & 715 & $\leq 0010$ & 047 & $323.41 \pm 148.53$ & $16011+4947$ & 2024 & $24<0001$ & 092 \\
\hline FIISt NiC) (L) & MT + E Group & $339.90 \pm 184.74$ & $397.97 \pm 172.87$ & $54.52 \pm 4.84$ & 4.10 & $j^{\prime}<0.048$ & 0.31 & $421.72 \pm 178.22$ & $81.79 \pm 6.81$ & 7.15 & $<0.010$ & 0.47 & $483.52 \pm 198.00$ & $160.11 \pm 49.47$ & 20.24 & $24<0.001$ & 0.92 \\
\hline$C_{2} 3(\mathrm{I})$ & E Group & $174.59 \pm 90.02$ & $185.90 \pm 80.34$ & $0286+0723$ & 717 & 7 & & $159.97 \pm 80.15$ & $118917+81=12$ & & -60001 & & $168.76 \pm 76.65$ & $16276+0020$ & & 50001 & 126 \\
\hline$(2-3(L)$ & MT + E Group & $206.38 \pm 113.72$ & $279.76 \pm 167.66$ & $93.86 \pm 87.32$ & 7.47 & $<0.008$ & 0.71 & $298.14 \pm 161.58$ & $138.17 \pm 81.43$ & 20.91 & $<0.001$ & 1.08 & $332.52 \pm 166.91$ & $163.76 \pm 90.26$ & 26.36 & $36<0.001$ & 1.26 \\
\hline Subccinital (I) & E Group & $180.59 \pm 79.85$ & $196.90 \pm 79.97$ & & & & & $181.90 \pm 67.35$ & $13201+890$ & & 50001 & 110 & $182.45 \pm 65.61$ & & & & 10 \\
\hline Suboccipitar (L) & MT + E Group & $207.90 \pm 105.33$ & $267.00 \pm 116.26$ & $0.10 \pm 36.29$ & 4.64 & $\begin{array}{l}y<0.036 \\
\end{array}$ & 0.70 & $314.14 \pm 155.73$ & $132.24 \pm 88.35$ & 16.92 & $<0.001$ & 1.10 & $380.66 \pm 198.09$ & $198.21 \pm 132.48$ & 28.06 & $6<<0.001$ & 1.34 \\
\hline
\end{tabular}

Abbreviature: E, Exercise; MT + E, Manual Therapy + Exercise; SD, Standard Deviation; +, most restricted or less range of movement between the two te

higher range of movement between the two test rotations; MCJ, First Metacarpal Joint; R, Right; L, Left; ${ }^{O A}$. One-way ANOVA; d. Cohen's d coefficient. 
The home exercise regime was performed every day (55.2\% MT + E and 65.5\% Exercise of participants), 4-6 days a week (31\% MT + E and 24.1\% Exercise), and 1-3 days a week (13.8\% MT + E and $10.3 \%$ Exercise). There were no differences between groups $(p>0.05)$.

\subsection{3-Month Follow Up (T2)}

In the within-group analysis (Table 2 ) of the exercise group, a statistically significant improvement was found in the craniocervical flexion test variable $(p<0.01)$. However, a statistically significant worsening in the flexion-rotation test to the less restricted side was found $(p<0.01)$. In the MT + E group, there was a statistically significant improvement in the visual analogue scale, upper cervical flexion, neck disability index, flexion-rotation test to the more $(+)$ and less $(-)$ restricted side $(p<0.05)$ and craniocervical flexion test $(p<0.01)$ (Figure 6). There was also a statistically significant improvement in the pressure pain threshold variables at C2-3 (right), suboccipital (right), C2-3 (left), and suboccipital (left) $(p<0.05)$.

In the between-group analysis (Table 3), statistically significant differences were found between both groups in favor of the MT + E group for the visual analogue scale, upper cervical flexion, neck disability index $(p<0.05)$, flexion-rotation test variables to the more $(+)$ and less $(-)$ restricted side $(p<0.01)$ and in the pressure pain threshold variables (first metacarpal joint (right), C2-3 (right), suboccipital (right), first metacarpal joint (left), C2-3 (left) and suboccipital variables (left)) $(p<0.05)$. Differences were also found in the GROC-Scale variable $(p<0.01)$ (Figure 7).

The home exercise regime was performed every day (31\% MT $+\mathrm{E}$ and $48.3 \%$ Exercise of participants), 4-6 days a week (20.7\% MT + E and 34.5\% Exercise), 1-3 days a week (31\% MT + E and $17.2 \%$ Exercise), and less than 1 day a week (17.2\% MT + E, and 0\% Exercise). Significant differences were found between groups in favor of the exercise group $(p<0.05)$.

\subsection{6-Month Follow Up (T3)}

In the within-group analysis (Table 2) of the exercise group, a statistically significant improvement was found in the craniocervical flexion test $(p<0.01)$. However, a statistically significant worsening in the flexion-rotation test to the less $(-)$ restricted side $(p<0.05)$ was observed. In the group MT $+\mathrm{E}$, statistically significant improvements were found in the visual analogue scale, upper cervical flexion, neck disability index, flexion-rotation test to the more $(+)$ and less $(-)$ restricted side, and craniocervical flexion test $(p<0.01)$ (Figure 6). A statistically significant improvement was found in the pressure pain threshold variables in C2-3 (right), suboccipital (right), first metacarpal joint (left), C2-3 (left), and suboccipital (left) $(p<0.01)$.

In the between-group analysis (Table 3), statistically significant differences were found between both groups in favor of the MT + E group in the visual analogue scale, upper cervical flexion, neck disability index, flexion-rotation test to the more $(+)$ and less $(-)$ restricted side $(p<0.01)$, in the pressure pain threshold variables (first metacarpal joint (right), C2-3 (right), suboccipital (right), first metacarpal joint (left), C2-3 (left), and suboccipital variables (left)), GROC-Scale $(p<0.01)$ (Figure 7), and in the craniocervical flexion test variable $(p<0.01)$ (Figure 6).

The home exercise regime was performed every day (10.3\% MT + E and 17.2\% Exercise of participants), 4-6 days a week (24.1\% MT + E and 51.7\% Exercise), 1-3 days a week (48.3\% MT + E and $31 \%$ Exercise, and less than 1 day a week (17.2\% MT + E and 0\% Exercise). The Exercise group performed the home exercise more regularly with statistical significance $(p<0.05)$.

\section{Discussion}

The objective of this study was to compare the short- and mid-term effectiveness of adding a manual therapy approach to a cervical exercise protocol in patients with chronic neck pain and upper cervical spine dysfunction. A statistically significant improvement in all study variables was found in the group that received manual therapy and exercise over the exercise group in the short- and mid-term. 
Both groups showed statistically significant improvements in the neck disability index at the end of the treatment period (T1). Several articles support the use of cervical exercises leading to positive results in patients' disabilities $[6,40,41,45]$. However, the improvement in the MT + E group was more than double that of the exercise group in our study and greater than those found in earlier studies $[4,12,46]$. At T2 and T3, only the MT + E group showed statistically significant improvements and showed a clinically relevant change (5 and 7 points, respectively) [47]. Similarly, patients' perception of improvement reflects a statistically significant greater improvement in the MT + E group compared to the exercise group using the GROC-Scale. Different studies consider that patient's perception is an important variable to take into account in a bio-psycho-social model [48-50].

Adding a manual therapy approach focused on treating upper cervical joint restriction of movement to a cervical exercise protocol significantly improved upper cervical mobility in the sagittal and transverse planes. The range of motion of the flexion-rotation test significantly improved after 1 month (T1), 3 months (T2), and 6 months (T3). At T2 and T3, upper cervical flexion also increased significantly. The flexion-rotation test measures the upper cervical rotation, mainly in C1-2 segment [26]. The manual therapy approach aimed to restore the mobility of the upper cervical joints by treating occipital-atlas (C0-1) and axis-C3 (C2-3) and then, if necessary, atlas-axis (C1-2) segment. Apart from treating directly C1-2 [8], the application of manual therapy in C0-1 and C2-3 [8,23,51,52] has been shown to improve the flexion rotation test. Our manual therapy approach followed international safety recommendations promoting the indirect treatment of the segment with more dysfunction (in this case, C1-2) and avoiding end-range procedures [19]. Our study suggests that adding manual therapy to a cervical exercise protocol could be beneficial for maintaining an upper cervical range of motion at a mid-term follow-up. Several studies have shown that this improvement is sustained over the long term for one to three years, even when the continuation of home exercises after initial treatment has been inconsistent $[37,53]$. However, unlike previous studies $[8,19,20]$, the improvement of the upper cervical range of motion was not present in our exercise group. Our specific inclusion criteria may explain this difference as patients with upper cervical spine restriction of movement could experience greater difficulty in improving the upper cervical range of motion while performing the cervical exercise [54].

In contrast, although there was a tendency to improve the performance in the MT + E group at T3, both groups showed a statistically significant improvement in the craniocervical flexion test in short- and mid-term follow-ups. The craniocervical flexion test measures the activation of the deep cervical flexor muscles. There are several studies that demonstrate the same result when applying manual therapy or MT $+\mathrm{E}$ techniques $[7,55,56]$ and exercises $[41,57]$. The rationale behind using manual therapy may be explained by the linear relationship between upper cervical spine range of motion and the contractile capacity of deep cervical musculature [58] and the improved recruitment in deep muscles and less activation in superficial muscles shown by the application a specific cervical mobilization [59].

Our study showed that adding a manual therapy approach focused on treating upper cervical joint restriction of movement to a cervical exercise protocol significantly diminished the visual analogue scale for pain and increased the pressure pain thresholds in all follow-ups. These results regarding pain alleviation are similar to the studies that found improvements in cervical pain in MT + E groups [7,60], although studies with an isolated manual therapy approach [12] and exercise [61] have also shown a reduction in cervical pain. Considering pressure pain threshold variables, previous studies did not identify any difference when applying manual therapy alone [23,62]. Our MT + E was more effective than the exercise group, showing similar results to Celenay et al., 2016 [5], although other authors found no difference between groups [41,46]. Similar to our results, different studies suggest that manual therapy provokes hypoalgesia locally and in regions distant from the area of the segmental treatment [63-67]. The underlying explanation for this hypoalgesia is the activation of segmental inhibitory pathways, spinal cord pathways, or descending inhibitory pathways of the brain stem $[68,69]$. 


\section{Limitations}

The study presents some limitations. The results of this study are limited to a sample presenting several inclusion and exclusion criteria, and only one therapist provided the treatment in the current study, which may limit the generalization of the results. The manual therapy approach was adapted to the clinical finding of the upper cervical spine. This clinical approach did not allow the determination of which specific intervention was more effective. In addition, the exercise protocol included a home exercise regime. Although subjects were periodically asked about and supervised for the performance of the home exercises, the methodology presented limitations in controlling the frequency and execution of the home exercises.

Active function testing of the cervical muscles was limited to the deep ventral flexors with the upper cervical flexion active range of motion and the craniocervical flexion test. Therefore, no generalization of the results to other cervical muscles could be stated. Another limitation is that the last follow-up was done after 6 months, so we cannot know the long-term evolution. Finally, even though patients were referred by doctors, the presence of neck pain with a visceral origin may have been underestimated, so our clinical trial may have taken into account visceral referred neck pain in the inclusion and exclusion criteria [70].

\section{Conclusions}

Adding manual therapy within a program of four 20-min sessions of exercise and home-exercise was found to be more effective than the same exercise program without including manual therapy, in patients with chronic neck pain and upper rotation restriction. Outcomes in neck disability index, patients' perception of improvement, upper cervical range of motion, pain intensity, and pressure pain thresholds improved in the short- (3-months) and mid-term (6-months) and craniocervical flexion test in the mid-term were better in patients who received manual therapy.

Author Contributions: J.R.-S., Concept development, design, data collection/processing, literature search and writing; M.M.-U., design, supervision, literature search and critical review; J.C.-d.-T., data collection, literature search, writing and critical review; C.L.-d.-C., analysis/interpretation of the results and critical review; M.O.L.-L., supervision, writing and critical review; J.M.T.-M., supervision and critical review; A.I.L., analysis/interpretation of the results and critical review; C.H.-G., concept development, design, supervision, literature search, writing and critical review. All authors have read and agreed to the published version of the manuscript.

Funding: This research did not receive any specific grant from funding agencies in the public, commercial, or not-for-profit sectors.

Acknowledgments: Thanks to all the patients who have participated in this study, as well as to the OMT-E Clinical Center of Zaragoza, the "Delicias Sur" Health Center of Zaragoza, and the Physiotherapy Research Unit of the University of Zaragoza.

Conflicts of Interest: The authors declare that there is no conflict of interest.

Data Availability: The datasets used and/or analyzed during the current study are available from the corresponding author on reasonable request.

\section{References}

1. Audette, I.; Dumas, J.-P.; Côté, J.N.; De Serres, S.J. Validity and between-day reliability of the Cervical Range of Motion (CROM) Device. J. Orthop. Sports Phys. Ther. 2010, 40, 318-323. [CrossRef] [PubMed]

2. Rodríguez-Sanz, J.; Carrasco, A.; Cabanillas-Barea, S.; Hidalgo-García, C.; Fanlo-Mazas, P.; Lucha-López, M.O.; Tricás-Moreno, J.M. Validity and reliability of two smartphone applications to measure the lower and upper cervical spine range of motion in subjects with chronic cervical pain. J. Back Musculoskelet. Rehabil. 2019, 32, 619-627. [CrossRef] [PubMed]

3. Hidalgo-García, C.; Tricás-Moreno, J.M.; Lucha-López, O.; Miguel, E.E.; Bueno-Gracia, E.; Pérez-Guillén, S.; Fanlo-Mazas, P.; Ruiz-De-Escudero, A.; Krauss, J. Short term efficacy of C0-C1 mobilization in the cervical neutral position in upper cervical hypomobility: A randomized controlled trial. J. Int. Acad. Phys. Ther. Res. 2016, 7, 908-914. [CrossRef] 
4. Kay, T.M.; Gross, A.; Goldsmith, C.H.; Hoving, J.L.; Brønfort, G. Exercises for mechanical neck disorders. Cochrane Database Syst. Rev. 2005, CD004250. [CrossRef]

5. Celenay, S.T.; Akbayrak, T.; Kaya, D.O. A comparison of the effects of stabilization exercises plus manual therapy to those of stabilization exercises alone in patients with nonspecific mechanical neck pain: A randomized clinical trial. J. Orthop. Sports Phys. Ther. 2016, 46, 44-55. [CrossRef]

6. Blomgren, J.; Strandell, E.; Jull, G.; Vikman, I.; Roijezon, U. Effects of deep cervical flexor training on impaired physiological functions associated with chronic neck pain: A systematic review. BMC Musculoskelet. Disord. 2018, 19, 415. [CrossRef]

7. Miller, J.; Gross, A.; D’Sylva, J.; Burnie, S.J.; Goldsmith, C.H.; Graham, N.; Haines, T.; Brønfort, G.; Hoving, J.L. Manual therapy and exercise for neck pain: A systematic review. Man. Ther. 2010, 15, 334-354. [CrossRef]

8. Lluch, E.; Schomacher, J.; Gizzi, L.; Petzke, F.; Seegar, D.; Falla, D. Immediate effects of active cranio-cervical flexion exercise versus passive mobilisation of the upper cervical spine on pain and performance on the cranio-cervical flexion test. Man. Ther. 2014, 19, 25-31. [CrossRef]

9. Aker, P.D.; Gross, A.R.; Goldsmith, C.H.; Peloso, P. Conservative management of mechanical neck pain: Systematic overview and meta-analysis. BMJ 1996, 313, 1291-1296. [CrossRef]

10. Beltran-Alacreu, H.; López-De-Uralde-Villanueva, I.; Fernández-Carnero, J.; La Touche, R. Manual therapy, therapeutic patient education, and therapeutic exercise, an effective multimodal treatment of nonspecific chronic neck pain. Am. J. Phys. Med. Rehabil. 2015, 94, 887-897. [CrossRef]

11. Dunning, J.R.; Cleland, J.A.; Waldrop, M.A.; Arnot, C.; Young, I.; Turner, M.; Sigurdsson, G. Upper cervical and upper thoracic thrust manipulation versus nonthrust mobilization in patients with mechanical neck pain: A multicenter randomized clinical trial. J. Orthop. Sports Phys. Ther. 2012, 42, 5-18. [CrossRef] [PubMed]

12. Pérez, H.I.; Perez, J.L.A.; Gil-Martínez, A.; La Touche, R.; Lara, S.L.; Gonzalez, N.C.; Perez, H.A.; Bishop, M.; Fernández-Carnero, J. Is one better than another? A randomized clinical trial of manual therapy for patients with chronic neck pain. Man. Ther. 2014, 19, 215-221. [CrossRef] [PubMed]

13. Hall, T.M.; Robinson, K. The flexion-rotation test and active cervical mobility-A comparative measurement study in cervicogenic headache. Man. Ther. 2004, 9, 197-202. [CrossRef] [PubMed]

14. Ogince, M.; Hall, T.; Robinson, K.; Blackmore, A.M. The diagnostic validity of the cervical flexion-rotation test in C1-C2 related cariogenic headache. Man Ther. 2007, 12, 256-269. [CrossRef]

15. Blanpied, P.R.; Gross, A.R.; Elliott, J.M.; Devanet, L.L.; Clewley, D.; Walton, D.; Sparks, C.; Robertson, E. Neck pain: Revision. Clinical practice guidelines linked to the international classification of functioning, disability, and health from the orthopaedic section of the American Physical Therapy Association. J. Orthop. Sport. Phys. Ther. 2016, 46, 1-67.

16. Zito, G.; Jull, G.; Story, I. Clinical tests of musculoskeletal dysfunction in the diagnosis of cervicogenic headache. Man. Ther. 2006, 11, 118-129. [CrossRef]

17. Kaltenborn, F. Manual Mobilization of the Joints. Volume II: The Spine; OMT España: Oslo, Norway, 2012.

18. Bier, J.D.; Scholten-Peeters, G.G.; Staal, J.B.; Pool, J.; Van Tulder, M.W.; Beekman, E.; Knoop, J.; Meerhoff, G.; Verhagen, A.P. Clinical practice guideline for physical therapy assessment and treatment in patients with nonspecific neck pain. Phys. Ther. 2017, 98, 162-171. [CrossRef]

19. Rushton, A.; Rivett, D.; Carlesso, L.; Flynn, T.; Hing, W.; Kerry, R. International framework for examination of the cervical region for potential of cervical arterial dysfunction prior to orthopaedic manual therapy intervention. Man. Ther. 2014, 19, 222-228. [CrossRef]

20. Rueda, V.G.; López-De-Celis, C.; Barra-López, M.; Uribarren, A.C.; Tomás, S.C.; Hidalgo-García, C. Effectiveness of a specific manual approach to the suboccipital region in patients with chronic mechanical neck pain and rotation deficit in the upper cervical spine: Study protocol for a randomized controlled trial. BMC Musculoskelet. Disord. 2017, 18, 384. [CrossRef]

21. Ortega, J.A.A.; Delgado-Martínez, A.D.; Ruiz, R.A. Validación de una versión española del Índice de Discapacidad Cervical. Med. Clínica 2008, 130, 85-89. [CrossRef]

22. Mawdsley, R.H.; Moran, K.A.; Conniff, L.A. Reliability of two commonly used pain scales with elderly patients. J. Geriatr. Phys. Ther. 2002, 25, 16-20. [CrossRef]

23. Malo-Urriés, M.; Tricás-Moreno, J.M.; Estébanez-De-Miguel, E.; Hidalgo-García, C.; Carrasco, A.; Cabanillas-Barea, S. Immediate effects of upper cervical translatoric mobilization on cervical mobility and pressure pain threshold in patients with cervicogenic headache: A randomized controlled trial. J. Manip. Physiol. Ther. 2017, 40, 649-658. [CrossRef] [PubMed] 
24. Hall, T.M.; Robinson, K.W.; Fujinawa, O.; Akasaka, K.; Pyne, E.A. Intertester reliability and diagnostic validity of the cervical flexion-rotation test. J. Manip. Physiol. Ther. 2008, 31, 293-300. [CrossRef]

25. Hall, T.M.; Briffa, K.; Hopper, D.; Robinson, K. Long-term stability and minimal detectable change of the cervical flexion-rotation test. J. Orthop. Sports Phys. Ther. 2010, 40, 225-229. [CrossRef] [PubMed]

26. Takasaki, H.; Hall, T.M.; Oshiro, S.; Kaneko, S.; Ikemoto, Y.; Jull, G. Normal kinematics of the upper cervical spine during the flexion-rotation test-In Vivo measurements using magnetic resonance imaging. Man. Ther. 2011, 16, 167-171. [CrossRef]

27. Strimpakos, N. The assessment of the cervical spine. Part 1: Range of motion and proprioception. J. Bodyw. Mov. Ther. 2011, 15, 114-124. [CrossRef]

28. Jull, G.; O'Leary, S.; Falla, D. Clinical assessment of the deep cervical flexor muscles: The craniocervical flexion test. J. Manip. Physiol. Ther. 2008, 31, 525-533. [CrossRef]

29. James, G.; Doe, T. The craniocervical flexion test: Intra-tester reliability in asymptomatic subjects. Physiother. Res. Int. 2010, 15, 144-149. [CrossRef]

30. Fischer, A.A. Pressure algometry over normal muscles. Standard values, validity and reproducibility of pressure threshold. Pain 1987, 30, 115-126. [CrossRef]

31. Chesterton, L.S.; Sim, J.; Wright, C.C.; Foster, N.E. Inter-rater reliability of algometry in measuring pressure pain thresholds in healthy humans, using multiple raters. Clin. J. Pain 2007, 23, 760-766. [CrossRef]

32. Ayerbe-Gracia, J.; Sousa-Casasnovas, P. Outcome assessment in lumbar spine surgery: The patient's perspective. Neurocirugía 2004, 15, 447-457. [CrossRef]

33. Sullivan, M.D. The new subjective medicine: Taking the patient's point of view on health care and health. Soc. Sci. Med. 2003, 56, 1595-1604. [CrossRef]

34. Gross, A.; Paquin, J.; Dupont, G.; Blanchette, S.; LaLonde, P.; Cristie, T.; Graham, N.; Kay, T.; Burnie, S.; Gelley, G.; et al. Exercises for mechanical neck disorders: A Cochrane review update. Man. Ther. 2016, 24, 25-45. [CrossRef] [PubMed]

35. Kamper, S.J.; Maher, C.G.; Mackay, G. Global rating of change scales: A review of strengths and weaknesses and considerations for design. J. Man. Manip. Ther. 2009, 17, 163-170. [CrossRef] [PubMed]

36. Fernández-de-las-Peñas, C.; Cleland, J.H.P. Síndromes Dolorosos en el Cuello y el Miembro Superior. Detección, Diagnóstico y Tratamiento Informados por la Evidencia; Elsevier: Churchill \& Livingstone, UK, 2013.

37. Jull, G.; Trott, P.; Potter, H.; Zito, G.; Niere, K.; Shirley, D.; Emberson, J.R.; Marschner, I.C.; Richardson, C. A randomized controlled trial of exercise and manipulative therapy for cervicogenic headache. Spine 2002, 27, 1835-1843. [CrossRef] [PubMed]

38. Celenay, S.T.; Kaya, D.O.; Akbayrak, T. Cervical and scapulothoracic stabilization exercises with and without connective tissue massage for chronic mechanical neck pain: A prospective, randomised controlled trial. Man. Ther. 2016, 21, 144-150. [CrossRef] [PubMed]

39. Hansen, I.R.; Søgaard, K.; Christensen, R.; Thomsen, B.; Manniche, C.; Juul-Kristensen, B. Neck exercises, physical and cognitive behavioural-graded activity as a treatment for adult whiplash patients with chronic neck pain: Design of a randomised controlled trial. BMC Musculoskelet. Disord. 2011, 12, 274. [CrossRef]

40. Falla, D.; Lindstrøm, R.; Rechter, L.; Boudreau, S.; Petzke, F. Effectiveness of an 8-week exercise programme on pain and specificity of neck muscle activity in patients with chronic neck pain: A randomized controlled study. Eur. J. Pain 2013, 17, 1517-1528. [CrossRef] [PubMed]

41. Gallego-Izquierdo, T.; Pecos-Martín, D.; Girbés, E.L.; Plaza-Manzano, G.; Caldentey, R.; Melãos, R.; Mariscal, D.; Falla, D. Comparison of cranio-cervical flexion training versus cervical proprioception training in patients with chronic neck pain: A randomized controlled clinical trial. J. Rehabil. Med. 2016, 48, 48-55. [CrossRef]

42. Kaltenborn, F. Fisioterapia Manual. Volumen III: Manipulación-Tracción de las Extremidades y la Columna; OMT España: Zaragoza, Spain, 2009.

43. Krauss, J.; Evjenth, O.C. Manipulación Vertebral Translatoria; OMT España: Zaragoza, Spain, 2009.

44. Cohen, J. Statistical Power Analysis for the Behavioral Sciences, 2nd ed.; Lawrence Erlbaum Associates: Hillsdale, NJ, USA, 1988.

45. Treleaven, J.; Peterson, G.; Ludvigsson, M.L.; Kammerlind, A.-S.; Peolsson, A. Balance, dizziness and proprioception in patients with chronic whiplash associated disorders complaining of dizziness: A prospective randomized study comparing three exercise programs. Man. Ther. 2016, 22, 122-130. [CrossRef] 
46. Cho, J.; Lee, E.; Lee, S. Upper thoracic spine mobilization and mobility exercise versus upper cervical spine mobilization and stabilization exercise in individuals with forward head posture: A randomized clinical trial. BMC Musculoskelet. Disord. 2017, 18, 525. [CrossRef] [PubMed]

47. MacDermid, J.C.; Walton, D.M.; Avery, S.; Blanchard, A.; Etruw, E.; McAlpine, C.; Goldsmith, C.H. Measurement properties of the neck disability index: A systematic review. J. Orthop. Sports Phys. Ther. 2009, 39, 400-C12. [CrossRef] [PubMed]

48. Llach, X.B.; Tudela, L.L. Reflexiones sobre la investigación de resultados en salud. Atención Primaria 2002, 30, 388-391. [CrossRef]

49. Higginson, I.J.; Carr, A.J. Measuring quality of life: Using quality of life measures in the clinical setting. BMJ 2001, 322, 1297-1300. [CrossRef] [PubMed]

50. Donabedian, A. The end results of health care: Ernest codman's contribution to quality assessment and beyond. Milbank Q. 1989, 67, 233. [CrossRef] [PubMed]

51. González Rueda, V. Efectividad del Abordaje Específico de la Región Suboccipital en Pacientes con Cervicalgia Mecánica Crónica con Déficit de Rotación Cervical Superior; Universidad de Zaragoza: Zaragoza, Spain, 2018.

52. Carrasco-Uribarren, A. Efectividad de un Protocolo de Manipulación Tracción en Posición de Reposo de la Columna Cervical Superior en Pacientes con Mareo Cervicogénico; Universidad de Zaragoza: Zaragoza, Spain, 2018.

53. Walker, M.J.; Boyles, R.E.; Young, B.A.; Strunce, J.B.; Garber, M.B.; Whitman, J.M.; Deyle, G.; Wainner, R.S. The effectiveness of manual physical therapy and exercise for mechanical neck pain. Spine 2008, 33, 2371-2378. [CrossRef] [PubMed]

54. Hidalgo-García, C.; Cabanillas-Barea, S.; Malo-Urriés, M.; Fanlo-Mazas, P.; Jiménez-Del-Barrio, S.; Uribarren, A.C. Estudio descriptivo de la relación entre la inclinación cervical y rotación cervical superior en sujetos con hipomovilidad cervical superior. Cuest. Fisioter. Rev. Univ. Inf. Investig. Fisioter. 2015, 44, 143-150.

55. Kim, D.-H.; Kim, S.-Y. Comparison of immediate effects of sling-based manual therapy on specific spine levels in subjects with neck pain and forward head posture: A randomized clinical trial. Disabil. Rehabil. 2019, 1-8, 1-8. [CrossRef]

56. Galindez-Ibarbengoetxea, X.; Chourraut, I.S.; Ramírez-Vélez, R.; Andersen, L.L.; González-Izal, M.; Crespo, A.J.; Crespo, M.I. Immediate effects of osteopathic treatment versus therapeutic exercise on patients with chronic cervical pain. Altern. Ther. Heal. Med. 2017, 24, 24-32.

57. Beer, A.; Treleaven, J.; Jull, G. Can a functional postural exercise improve performance in the cranio-cervical flexion test? A preliminary study. Man. Ther. 2012, 17, 219-224. [CrossRef]

58. Falla, D.; Campbell, C.D.; Fagan, A.E.; Thompson, D.C.; Jull, G. Relationship between cranio-cervical flexion range of motion and pressure change during the cranio-cervical flexion test. Man. Ther. 2003, 8, 92-96. [CrossRef]

59. De Jesus-Moraleida, F.R.; Ferreira, P.H.; Pereira, L.S.; Vasconcelos, C.M.; Ferreira, M.L. Ultrasonographic analysis of the neck flexor muscles in patients with chronic neck pain and changes after cervical spine mobilization. J. Manip. Physiol. Ther. 2011, 34, 514-524. [CrossRef] [PubMed]

60. Farooq, M.N.; Mohseni-Bandpei, M.A.; Gilani, S.A.; Ashfaq, M.; Mahmood, Q. The effects of neck mobilization in patients with chronic neck pain: A randomized controlled trial. J. Bodyw. Mov. Ther. 2018, 22, $24-31$. [CrossRef] [PubMed]

61. Kim, S.-H.; Choi, J.-H.; Lee, K.-W. Immediate effects of active stretching versus passive mobilization of the upper cervical spine on patients with neck pain and ROM. J. Korean Soc. Phys. Med. 2016, 11, $27-32$. [CrossRef]

62. River, Y.; Levital, T.; Belgrade, M. Computerized mobilization of the cervical spine for the treatment of chronic neck pain. Clin. J. Pain 2012, 28, 790-796. [CrossRef]

63. Wright, A. Hypoalgesia post-manipulative therapy: A review of a potential neurophysiological mechanism. Man. Ther. 1995, 1, 11-16. [CrossRef]

64. Grant, R.; Exelby, L. Physical therapy of the cervical and thoracic spine (clinics in physical therapy). Physiotherapy 1994, 80, 870. [CrossRef]

65. Vicenzino, B.; Collins, D.; Wright, A. The initial effects of a cervical spine manipulative physiotherapy treatment on the pain and dysfunction of lateral epicondylalgia. Pain 1996, 68, 69-74. [CrossRef]

66. Pickar, J.G. Neurophysiological effects of spinal manipulation. Spine J. 2002, 2, 357-371. [CrossRef]

67. Haas, M.; Groupp, E.; Panzer, D.; Partna, L.; Lumsden, S.; Aickin, M. Efficacy of cervical endplay assessment as an indicator for spinal manipulation. Spine 2003, 28, 1091-1096. [CrossRef] 
68. Vicenzino, B.; Paungmali, A.; Buratowski, S.; Wright, A. Specific manipulative therapy treatment for chronic lateral epicondylalgia produces uniquely characteristic hypoalgesia. Man. Ther. 2001, 6, 205-212. [CrossRef] [PubMed]

69. Skyba, A.D.; Radhakrishnan, R.; Rohlwing, J.J.; Wright, A.; Sluka, A.K.; Skyba, D.; Sluka, K. Joint manipulation reduces hyperalgesia by activation of monoamine receptors but not opioid or GABA receptors in the spinal cord. Pain 2003, 106, 159-168. [CrossRef]

70. Oliva-Pascual-Vaca, Á.; González-González, C.; Oliva-Pascual-Vaca, J.; Piña-Pozo, F.; Ferragut-Garcías, A.; Fernández-Domínguez, J.C.; Heredia-Rizo, A.M. Visceral Origin: An Underestimated Source of Neck Pain. A Systematic Scoping Review. Diagnostics 2019, 9, 186. [CrossRef] [PubMed]

(C) 2020 by the authors. Licensee MDPI, Basel, Switzerland. This article is an open access article distributed under the terms and conditions of the Creative Commons Attribution (CC BY) license (http://creativecommons.org/licenses/by/4.0/). 